\title{
Selection Criteria and Analysis of LCC Resonant DC - DC Converters for Automotive Applications
}

\author{
M. Prabhakar (Corresponding Author) \\ Department of EEE, Sri Venkateswara College of Engineering \\ Sriperumbudur, India - 602105 \\ E-mail: prab@svce.ac.in \\ S. Arulmozhi \\ Department of EEE, Sri Venkateswara College of Engineering \\ Sriperumbudur, India - 602105 \\ E-mail: arulmozhi@svce.ac.in \\ Dr. V. Kamaraj \\ Department of EEE, SSN College of Engineering \\ Kalavakkam, India - 603110 \\ E-mail: kamarajv@ssn.edu.in
}

\begin{abstract}
In this paper, a technique is developed for the selection of LCC topologies for automotive applications. All available LCC topologies are categorised based on the order of their voltage gain expression. Source/sink requirement for automotive applications is specified based on which some topologies are found to be realizable. Voltage gain expressions for these topologies are obtained and plotted. From the voltage gain plot, two candidate topologies are chosen. Closed form expressions for current gain, stress across resonant tank elements, circulating reactive power and stored energy in the tank are obtained. In addition, the optimum value of $\mathrm{Q}$ which results in smallest possible resonant tank is determined and is found to be 0.545 .
\end{abstract}

Keywords: Automotive applications, DC - DC converters, Resonant converters

\section{Nomenclature}

L Inductance value of resonant inductor L.

$\mathrm{C}_{1}, \mathrm{C}_{2} \quad$ Capacitance value of resonant capacitors $\mathrm{C}_{1}, \mathrm{C}_{2}$.

$\mathrm{X} \quad$ Ratio of capacitors.

Q Loaded Q value of the resonant circuit.

$\mathrm{R}_{\mathrm{L}} \quad$ Load resistance.

$\mathrm{R}_{\mathrm{eq}} \quad$ AC equivalent of load resistance.

$Z_{n} \quad$ Characteristic impedance of the resonant circuit.

$\mathrm{Z}_{\mathrm{in}} \quad$ Input impedance of the resonant circuit.

$\omega \quad$ Angular switching frequency in radians per second.

$\omega_{0} \quad$ Angular resonant frequency in radians per second.

$\omega_{n} \quad$ Normalised switching frequency. 


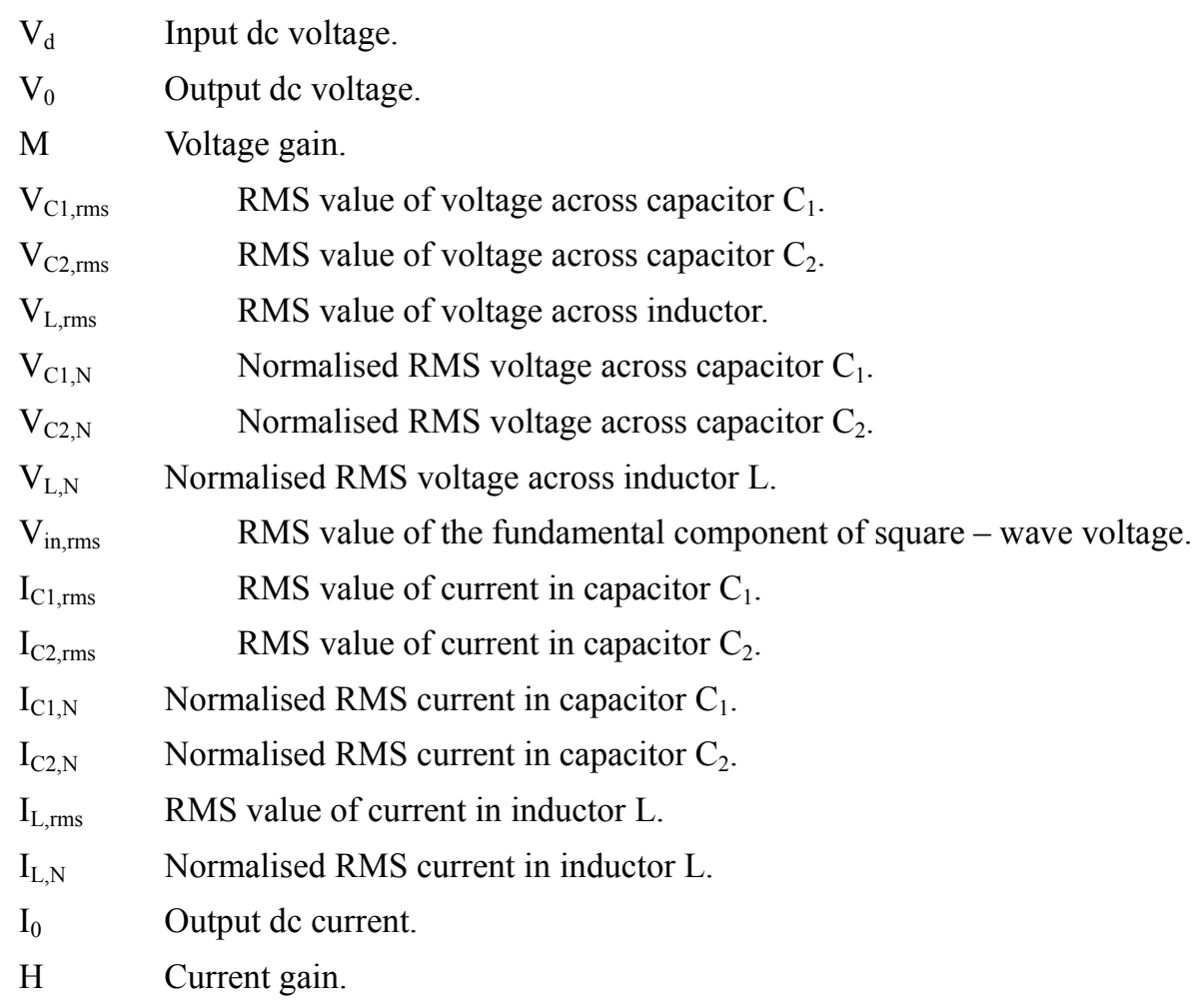

\section{Introduction}

Resonant converters possess several desirable features like zero voltage switching (ZVS), zero current switching (ZCS), high frequency operation, high efficiency, small size and low electromagnetic interference (EMI). They have been successfully applied to dc power supplies for industrial, commercial and domestic applications. Due to the sinusoidal behavior of resonant converters, their switching losses are much reduced. Therefore, it is possible to operate these converters at high frequencies and thus reduce the size of their reactive components. Consequently, several of today's resonant DC - DC converter operates in megahertz frequency range (Robert L. Steigerwarld, 1984, Forsyth A.J, 1996). The series and parallel resonant converters are basic resonant converter topologies. Generally, the resonant tank consists of only two energy storage elements. Compared with the conventional second order resonant converters, higher order converters are shown to possess more desirable characteristics.

Resonant converter topologies in which the tank circuit consists of more than two or three energy storage elements have been reported in the open literature. A generalized analysis for the third order resonant converter based on sinusoidal approximation was presented and the investigation was limited to six topological "schemes" (Rudolf P. Severns, 1992). Investigation of three and four energy storage elements was reported and analysis of few topologies of the third order circuit was given (Issa Batarseh, 1994). The operation of LCL - T resonant DC - DC converter as a constant current power supply at a particular operating point was analysed (Mangesh Borage, 2005). However, most of the third order LCC topologies are not yet thoroughly analysed particularly for their suitability in automotive applications.

At present, some DC - DC converter topologies have been employed for automotive applications (Shaotang Chena, 1995, Zhenyue Hong, 2000, Itsda Boonyaroonate, 2002, Huang-Jen, 2006, Fang Z. Peng, 2003). The performance of some DC - DC converters for automotive applications has been compared (R.M.Schupbach, 2003). However, these converters were designed for an electric vehicle and hence fed the main drive motor only.

The objective of this paper is to analyse all possible third order LCC resonant converters for their suitability in automotive loads like wind shield wiper motor, head lamp, etc. Based on the desired voltage gain characteristic plot, two LCC topologies are analysed in detail. Closed form expressions for $\mathrm{kVA} / \mathrm{kW}$ and energy stored in the resonant are obtained. From these expressions, the optimum value of Q which gives smallest possible resonant tank is determined.

\section{Selection of topologies}

\subsection{Determination of order of resonant tank}

Figure 1 shows a generalized representation of the resonant converter. The order of the converter is defined by the order of its commutational network. In case of one inductor and two capacitors (LCC) topology, 18 network combinations are possible (Issa Batarseh, 1994). Figure 2 shows all the possible network combinations.

The voltage gain of the system is determined from the resonant tank transfer function. All the LCC resonant topologies 
shown in Figure 2 can be categorized based on the order of the resonant tank transfer function. The order is determined based on the following criteria:

(i) The order of the system is determined by the number of elements that contribute to dynamical change in energy.

(ii) Elements connected in shunt with the source and load does not contribute to the dynamical change in energy. Hence, the voltage gain (order) of such systems will always be unity.

(iii) A loop formed by two elements in which there is no possibility of dynamical change in energy between them, can be combined as a single element. When a possibility of dynamical energy change between this element and another element exists, then the order of the system will be 2 .

Based on the above criteria, 18 possible LCC topologies are classified as unit gain, first, second and third order systems as shown in Table 1.

\subsection{Choice of topology based on source/sink combination}

The resonant tank can be excited from either a voltage (v) or current (i) source and can be used to feed either a voltage or current sink. Thus, two individual source and sink combinations are possible. These two individual combinations can be suitably arranged to give four possible source/sink combinations: $\mathrm{v} / \mathrm{v}, \mathrm{v} / \mathrm{i}, \mathrm{i} / \mathrm{i}$ and $\mathrm{i} / \mathrm{v}$.

In automotive applications, the input power is fed from the battery. The converter output should exhibit good voltage regulation. Hence, the converter which is used for automotive application should be of $\mathrm{v} / \mathrm{v}$ type source/sink combination.

\subsection{Realisation of topologies for automotive applications}

For a particular LCC topology to be realizable, the following conditions should be satisfied: (a) Loop containing capacitor(s) and voltage source or sink should not be formed. (b) Cutest containing inductor(s) and a current source or sink should not be formed.

This is because when the converter is driven or terminated by a two state square wave voltage source or sink respectively, when the voltage changes its state instantly, an infinite current spike will occur in the capacitor. Similarly, when the converter is driven or terminated by a two state current source or sink respectively, when the source or sink current changes state instantly, an infinite voltage spike will occur across the inductance.

Based on these conditions, only 8 topologies which are shown in Figure 3 are realizable for automotive application. Voltage gain for all the 8 topologies are obtained using AC analysis as explained in the next section.

\section{Analysis of topologies}

\subsection{Introduction to AC analysis}

In the ac analysis, the output rectifier and the filter are replaced by the equivalent ac resistance and the square-wave input voltage source is replaced by its fundamental sinusoidal equivalent. The power transfer from input to output is assumed to be only via the fundamental component and the contribution of all the harmonics is neglected (Robert $\mathrm{L}$ Steigerwarld, 1988, Robert W. Erickson, 2001).

The equivalent ac resistance for the rectifier with capacitive filter and the RMS value of the fundamental component of square-wave voltage (input to resonant tank) are given by

$$
R_{a c}=\frac{\pi^{2}}{8} R_{L} \text { and } V_{i n, r m s}=\frac{2 \sqrt{2}}{\pi} V_{d}
$$

The resonant frequency and the normalised switching frequency is defined as

$$
\omega_{0}=\frac{1}{\sqrt{L C}} \text { and } \omega_{n}=\frac{\omega}{\omega_{0}}
$$

The characteristic impedance and Q of the resonant network is

$$
Z_{n}=\sqrt{\frac{L}{C}} \text { and } Q=\frac{\omega_{0} L}{R_{L}}=\frac{Z_{n}}{R_{L}}
$$

The voltage and current gain is defined as

$$
M=\frac{V_{0}}{V_{d}} \text { and } H=\frac{I_{0}}{V_{d} / Z_{n}}=M Q
$$

The voltage and current base values are given by

$V_{b}=V_{d}$ and $I_{b}=I_{\text {in }}=\frac{V_{d}}{Z_{\text {in }}}$

Voltage gain for all candidate topologies is computed by using equations (1) - (5). From the voltage gain expression, a 
characteristic plot is obtained for each topology. This is compared with the desired characteristic plot to decide upon the suitability for automotive applications. Table 2 gives the voltage gain expression for all candidate topologies.

\subsection{Desired voltage gain plot}

For automotive applications, fixed frequency operation over wide load ranges is preferred. This will ensure open loop mode of operation of the converter and thus results in simple configuration. In addition, it is desirable if the converter has good load regulation. This means that the output voltage or in turn the voltage gain needs to remain a constant at any one normalised frequency for various values of $Q$. Figure 4 shows the nature of desired voltage gain characteristics.

\subsection{Topology selection based on voltage gain plot}

Voltage gain plots for all the 8 topologies are plotted. Only two topologies, 1 and 2, meet the desired requirement. Figure 5 and Figure 6 shows the voltage gain plots for 1 and 2 respectively. Other topologies do not give the desired voltage gain characteristic.

For instance, in topology 6, voltage gain converges to zero at a normalised frequency of 1 . This feature is not desirable as the output voltage would not be sufficient enough to meet the load requirements. For topology 8 , voltage gain never remains a constant when loaded $\mathrm{Q}$ is varied. In order to obtain desired voltage gain, switching frequency has to be changed in accordance with load variation. Hence, it cannot be used in automotive application without using complicated closed loop control to change the frequency to obtain the required voltage gain. Similar justification can be applied to remaining topologies also. Figure 7 and Figure 8 shows the voltage gain plots for topology 6 and 8 respectively.

\subsection{Stresses across resonant elements}

To design a resonant converter, the voltage and current stress that the resonant elements are subjected must be known. The voltage and current stresses are derived from circuit theory basics. Table 3 and Table 4 gives expressions for normalised voltage and current stresses experienced by resonant elements $L, C_{1}$ and $C_{2}$ for topology 1 and 2 respectively. The constants $\mathrm{A}$ and $\mathrm{B}$ are defined in (6) and (7).

$$
\begin{aligned}
& A=\left(1+j \frac{\pi^{2} \omega_{n} x}{8 Q}-\omega_{n}^{2}(1+x)-j \frac{\pi^{2} \omega_{n}^{3} x}{8 Q}\right) \frac{2 \sqrt{2}}{\pi} \\
& B=1-\frac{1}{\omega_{n}^{2}}+j \frac{8 Q}{\pi^{2} \omega_{n} x}\left[\frac{1}{\omega_{n}^{2}}-(1+x)\right]
\end{aligned}
$$

\section{Optimization of resonant tank size}

The size of the resonant tank depends on the energy stored in the tank elements and the $(\mathrm{kVA} / \mathrm{kW})$ ratio. In order to obtain smallest possible resonant tank, the energy stored in resonant tank and $(\mathrm{kVA} / \mathrm{kW})$ ratio has to be minimum at a particular value of loaded quality factor $\mathrm{Q}$. The energy and $(\mathrm{kVA} / \mathrm{kW})$ ratio are computed from the stress equations. These parameters are plotted with respect to $\mathrm{Q}$. From the plot, the value of $\mathrm{Q}$ which gives smallest possible resonant tank is determined.

From the voltage gain plot, it is observed that the voltage gain for both topologies remain constant at $\omega_{n}=0.7$. Hence, the energy and $(\mathrm{kVA} / \mathrm{kW})$ ratio are computed at this operating point. The expressions for these parameters are given by (8) - (11) for topologies 1 and 2.

$E_{1}=\frac{\pi^{2}}{16 Q} \frac{0.00375 \frac{\pi^{4}}{Q^{2}}+0.0218 \frac{\pi^{2}}{Q^{2}}+0.028}{0.00199 \frac{\pi^{4}}{Q^{2}}+0.0004}$

$E_{2}=\frac{0.45 Q\left(0.0092 Q^{4}+4.5171 Q^{2}+3.363\right)}{Q^{2}(1.063+0.0022 Q)^{2}}$

$\left(\frac{k V A}{k W}\right)_{1}=\frac{\pi^{2}}{8 Q}\left[\frac{0.127 \frac{\pi^{2}}{8 Q}-j\left(0.03+0.072\left(\frac{\pi^{2}}{8 Q}\right)^{2}\right)}{\left(j 0.7 \frac{\pi^{2}}{8 Q}\right)^{2}}\right]$ 
$\left(\frac{k V A}{k W}\right)_{2}=\left[\frac{0.166 Q-j 0.061 \frac{8 Q^{2}}{\pi^{2}}+j 1.486 \frac{\pi^{2}}{8}}{Q}\right]$

Figure 9 and Figure 10 shows the energy and $(\mathrm{kVA} / \mathrm{kW})$ ratio plots respectively. It is observed that at $\mathrm{Q}=0.545$, these parameters are minimum. Thus, the size of the resonant tank is expected to be minimum when $Q=0.545$. The resonant tank elements have to be designed suitably.

\section{Conclusion}

In this paper, selection of LCC topologies based on order of the resonant tank network is discussed in detail. The existence of various orders of resonant tank is justified. Similar arguments as mentioned in this paper can be used to select other possible multi element topologies and determine the order of their voltage gain expression also. The voltage gain requirement for automotive application is specified and the realizable topologies are further short listed and analysed. Only 1 and 2 gives load-independent voltage gain of 2 and 1.5 respectively at $\omega_{n}=0.7$. Analysis of these two realizable topologies is presented in detail. Closed form expressions for various performance parameters like stresses across resonant tank elements, energy stored in the resonant tank and $(\mathrm{kVA} / \mathrm{kW})$ ratio are obtained. Based on these parameters, the size of the resonant tank is optimized. The value of $Q$ which results in minimum tank size is computed and found to be 0.545 . It is expected that this would help in designing the DC - DC converter with a smaller resonant tank and thus fulfill the high power density requirement.

\section{Acknowledgment}

The authors with to thank the management and staff of Sri Venkateswara College of Engineering, Sriperumbudur and SSN College of Engineering, Kalavakkam for providing necessary encouragement and support while carrying out this work.

\section{References}

Fang Z., Peng, Fan, Zhang, and Zhaoming, Qian. (2003). A magnetic - less DC-DC converter for dual voltage automotive systems, IEEE Transactions on Industry Applications, 39: 511-518.

Forsyth A.J. (1996). Review of resonant techniques in power electronic systems, Power Engineering Journal, 110-120.

Huang-Jen, Chiu and Li-Wei, Lin. (2006). A bidirectional DC - DC converter for fuel cell electric vehicle driving sytem, IEEE Transactions on Power Electronics, 21: 950-958.

Issa Batarseh. (1994). Resonant converter topologies with three and four Energy storage elements, IEEE Transactions on Power Electronics, 9: 64-73.

Itsda Boonyaroonate and Shinsaku Mori. (2002), A compact DC/AC inverter for automotive application, Proc. of IEEE Intl. Symposium on Circuits and Systems, 5: 829-832.

Mangesh Borage, Sunil Tiwari and Swarna Kotaiah. (2005). Analysis of LCL-T resonant converter as a constant current power supply, IEEE Transactions on Industrial Electronics, 52: 1547-1554.

R.M. Schupbach and J.C. Balda. (2003). Comparing DC - DC converters for power management in hybrid electric vehicles, Proc. of IEMDC, 3: 1369-1374.

Robert L. Steigerwald. (1984). High frequency resonant transistor DC - DC converters, IEEE Transactions on Industrial Electronics, 31: 181-191.

Robert L. Steigerwald. (1988). A comparison of half - bridge resonant converter topologies, IEEE Transactions on Power Electronics, 3: 174-182.

Robert W. Erickson, Dragon Maksimovic. (2001). Fundamentals of power electronics, $2^{\text {nd }}$ ed., Springer International, 705-726.

Rudolf P.Severns. (1992). Topologies for three element converters, IEEE Transactions on Power Electronics, 7: 89-98.

Shaotang Chena and Thomas A. Lipo. (1995). Soft - switched inverter for electric vehicle drives, Proc. of APEC 1995, 2: $586-591$.

Zhenyue Hong, Richard Duke and Simon Round. (2000). A resonant DC link inverter for an electric vehicle, Journal of Electrical and Electronics Engineering, 21: 65-71. 
Table 1. Topology categorisation based on order

\begin{tabular}{|l|l|l|l|l|}
\hline Order & $\begin{array}{l}\text { Unity } \\
\text { gain }\end{array}$ & $1^{\text {st }}$ order & $2^{\text {nd }}$ order & $3^{\text {rd }}$ order \\
\hline Topology Number & 7,18 & $8,11,16$ & $3,9,10,14,15,17$ & $1,2,4,5,6,12,13$ \\
\hline
\end{tabular}

Table 2. Voltage gain expression for all topologies

\begin{tabular}{|c|c|}
\hline Topology & Voltage gain \\
\hline 1 & $M=\frac{j \frac{\pi^{2} \omega_{n} x}{8 Q}}{1-\omega_{n}^{2}(1+x)+j \frac{\pi^{2} \omega_{n} x}{8 Q}\left(1-\omega_{n}^{2}\right)}$ \\
\hline 2 & $M=\frac{1}{1-\frac{1}{\omega_{n}^{2}}+j \frac{8 Q}{\pi^{2} \omega_{n} x}\left[\frac{1}{\omega_{n}^{2}}-(1+x)\right]}$ \\
\hline 3 & $M=\frac{j \frac{\pi^{2} \omega_{n}}{8 Q}}{1+j \frac{\pi^{2} \omega_{n}}{8 Q}(1+x)-\omega_{n}^{2}(1+x)}$ \\
\hline 4 & $M=\frac{j \frac{\pi^{2} \omega_{n}}{8 Q}\left(1+x-\omega_{n}^{2} x\right)}{1-\omega_{n}^{2}+j \frac{\pi^{2} \omega_{n}}{8 Q}\left(1+x-\omega_{n}^{2} x\right)}$ \\
\hline 5 & $M=\frac{j \frac{\pi^{2} \omega_{n}}{8 Q}\left(1-\omega_{n}^{2} x\right)}{1-\omega_{n}^{2}(1+x)+j \frac{\pi^{2} \omega_{n}}{8 Q}\left(1-\omega_{n}^{2} x\right)}$ \\
\hline 6 & $M=\frac{j \frac{\pi^{2} \omega_{n}}{8 Q}\left(1-\omega_{n}^{2} x\right)}{1-\omega_{n}^{2} x+j \frac{\pi^{2} \omega_{n}}{8 Q}\left(1+x-\omega_{n}^{2} x\right)}$ \\
\hline 7 & 1 \\
\hline 8 & $M=\frac{1}{1-j \frac{8 Q}{\pi^{2} \omega_{n} x}}$ \\
\hline
\end{tabular}


Table 3. Voltage and current stresses of topology 1

\begin{tabular}{|c|c|c|}
\hline Element & Voltage Stress & Current Stress \\
\hline $\mathrm{L}$ & $\frac{-\omega_{n}^{2}(1+x)-j \frac{\pi^{2} \omega_{n}^{3} x}{8 Q}}{A}$ & $\frac{j \omega_{n}(1+x)-\frac{\pi^{2} \omega_{n}^{2} x}{8 Q}}{A}$ \\
\hline $\mathrm{C}_{1}$ & $\frac{1+j \frac{\pi^{2} \omega_{n} x}{8 Q}}{A}$ & $\frac{j \omega_{n}-\frac{\pi^{2} \omega_{n}^{2} x}{8 Q}}{A}$ \\
\hline $\mathrm{C}_{2}$ & $\frac{1}{A}$ & $\frac{j \omega_{n} x}{A}$ \\
\hline
\end{tabular}

Table 4. Voltage and current stresses of topology 2

\begin{tabular}{|c|c|c|}
\hline Element & Voltage Stress & Current Stress \\
\hline $\mathrm{L}$ & $\frac{\pi}{2 \sqrt{2}}\left[\frac{1-j \frac{8 Q}{\pi^{2} \omega_{n} x}}{B}\right]$ & $\left.\frac{2 \sqrt{2}}{\pi} Q\left[\frac{\omega_{n}^{2} x}{8}\right] \frac{\pi^{2}}{8 \omega_{n} Q}\right]$ \\
\hline $\mathrm{C}_{1}$ & $\left.\frac{\pi}{2 \sqrt{2}}\left[\frac{\omega_{n}^{2}}{\pi^{2}}\right] \frac{8 Q}{\pi^{2}}\left(1+\frac{1}{\omega_{n}^{2} x}\right)\right]$ & $\frac{2 \sqrt{2}}{\pi} Q\left[\frac{1-\frac{1}{\omega_{n}^{2} x}-j \frac{\pi^{2}}{8 \omega_{n} Q}}{B}\right]$ \\
\hline $\mathrm{C}_{2}$ & $\frac{2 \sqrt{2} Q}{\pi \omega_{n} x}\left[\frac{1}{B}\right]$ & $\frac{2 \sqrt{2}}{\pi} \frac{Q}{B}$ \\
\hline
\end{tabular}

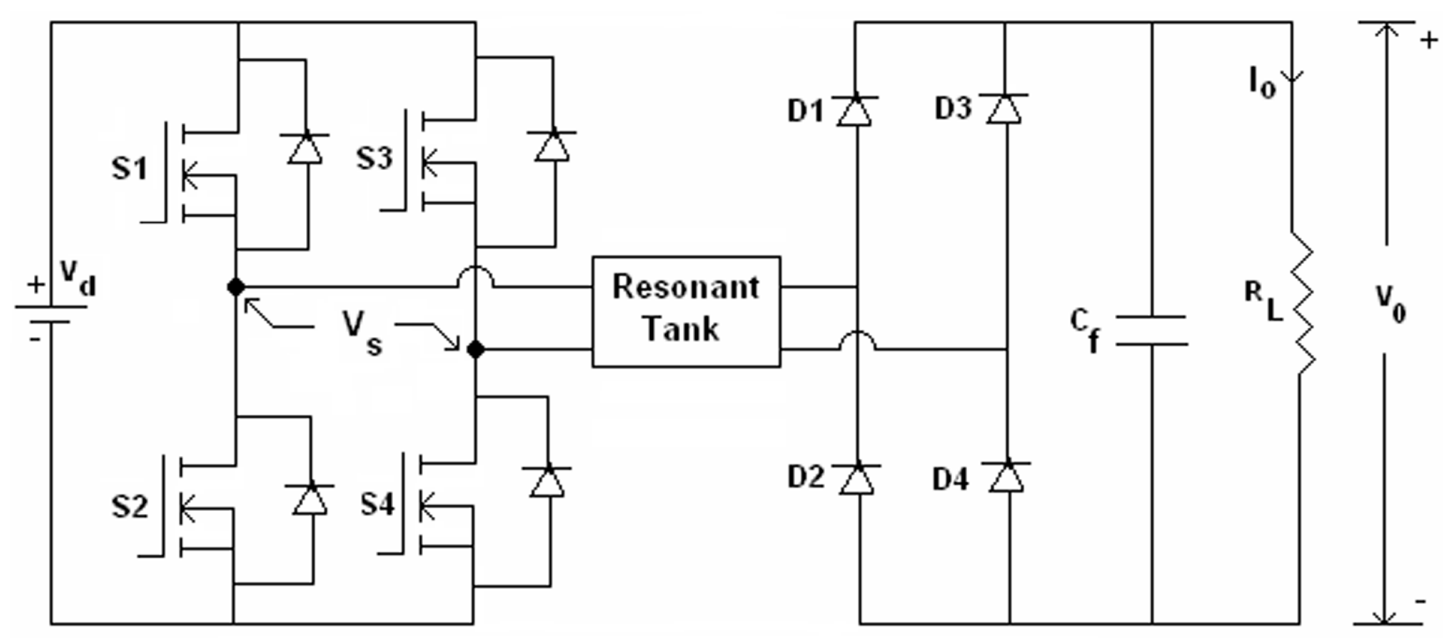

Figure 1. Generalised representation of a resonant converter 


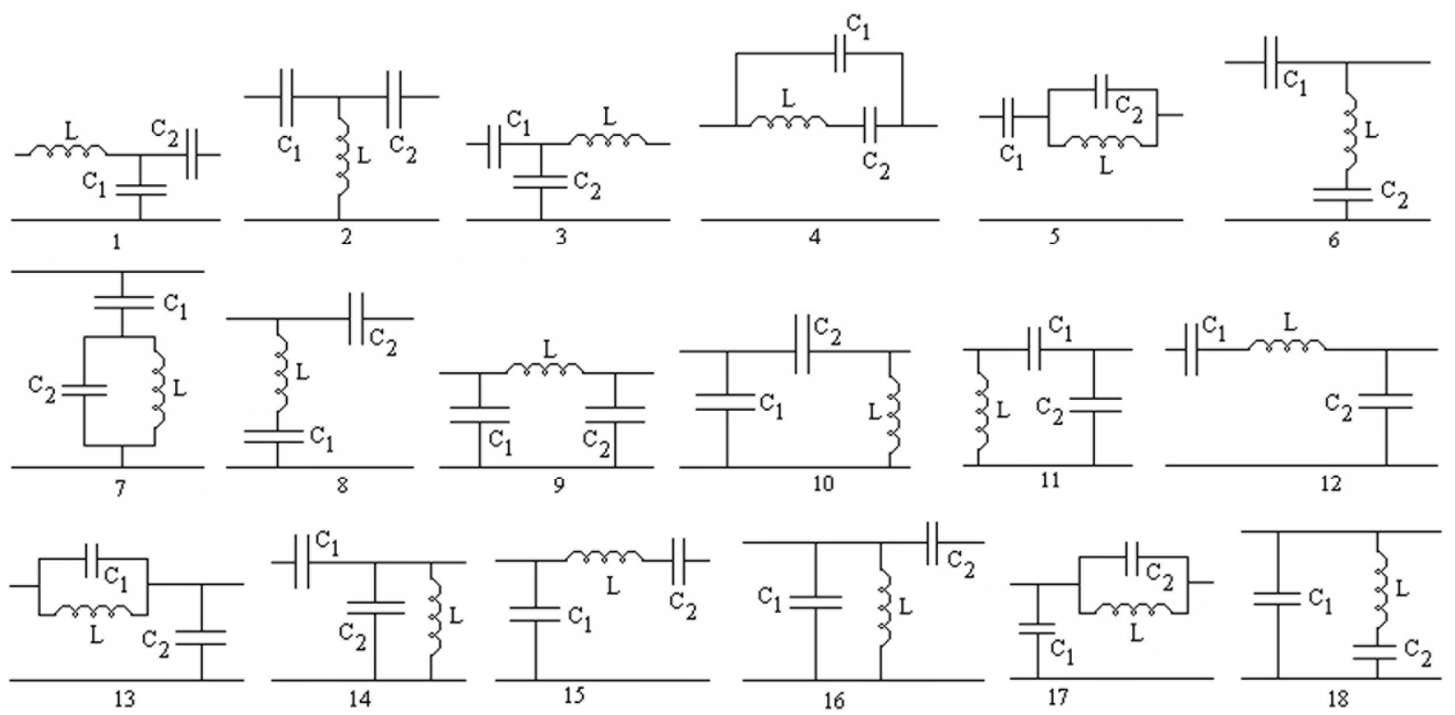

Figure 2. All possible LCC topologies

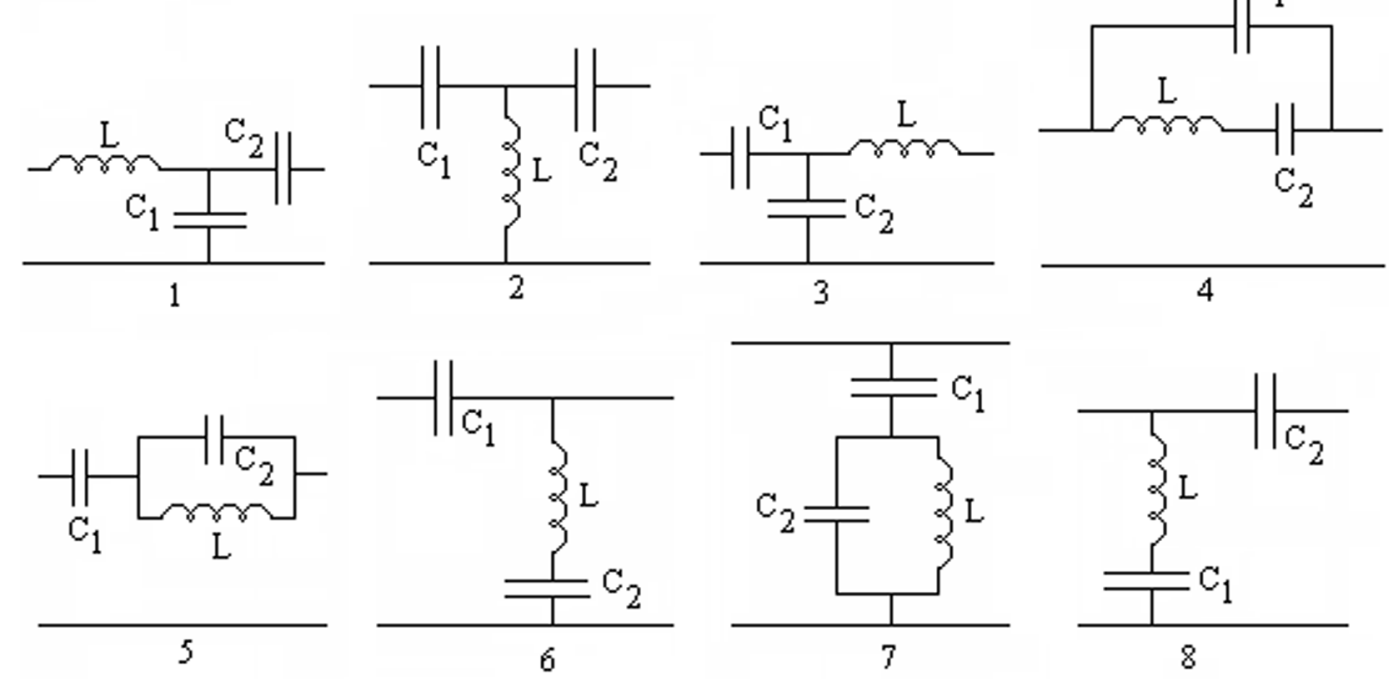

Figure 3. Realisable topologies

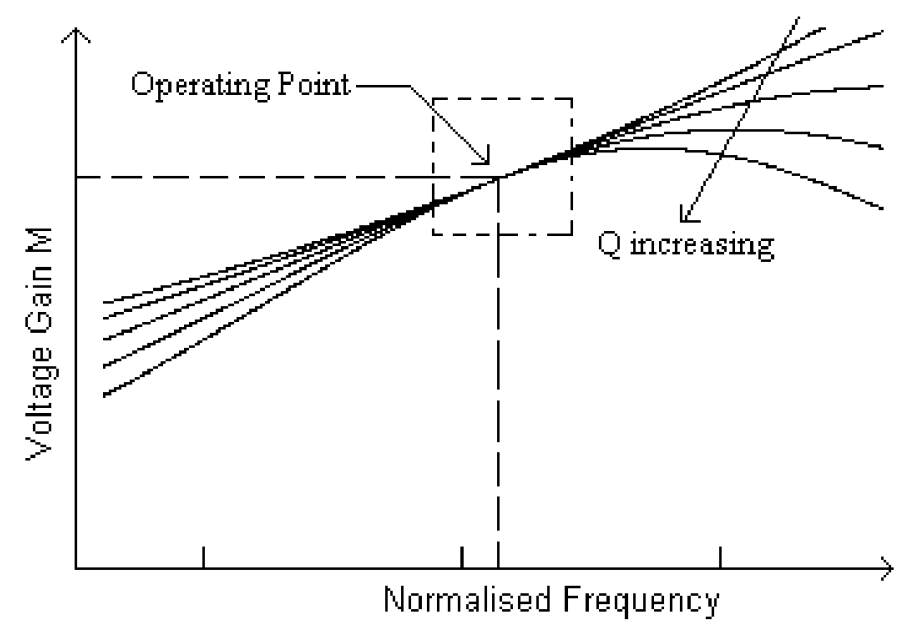

Figure 4. Desired voltage gain plot 


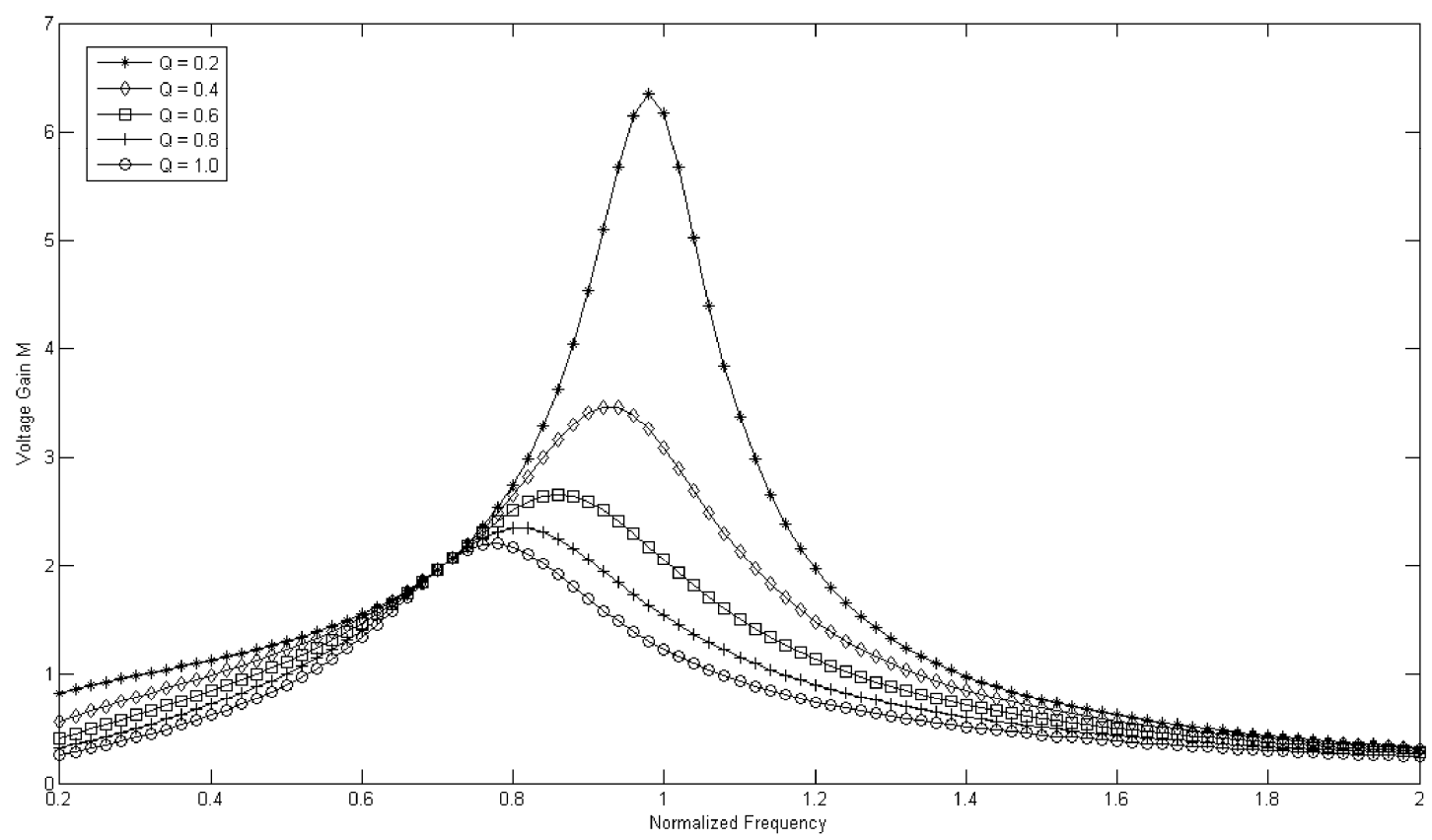

Figure 5. Voltage gain plot of topology 1

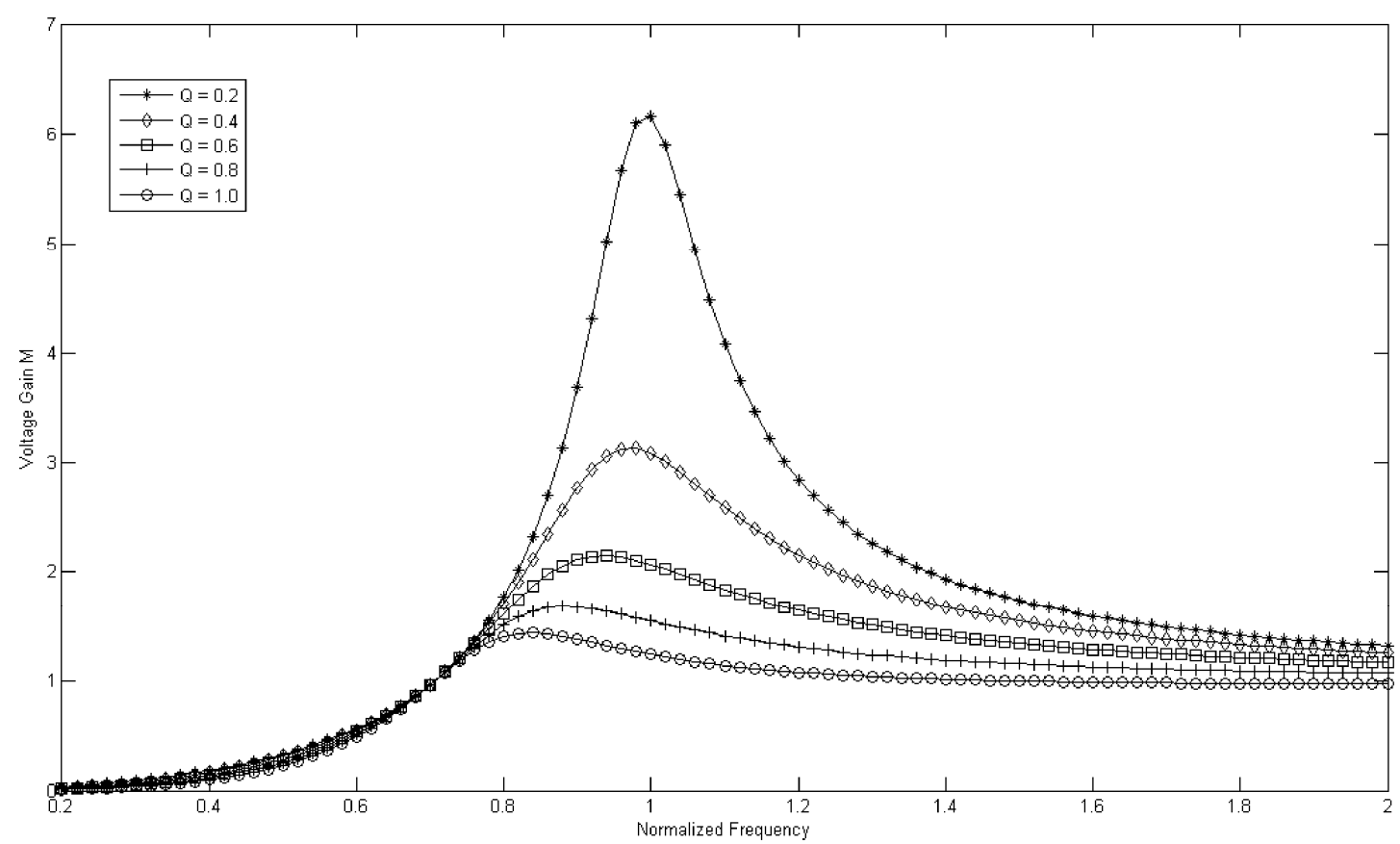

Figure 6. Voltage gain plot of topology 2 


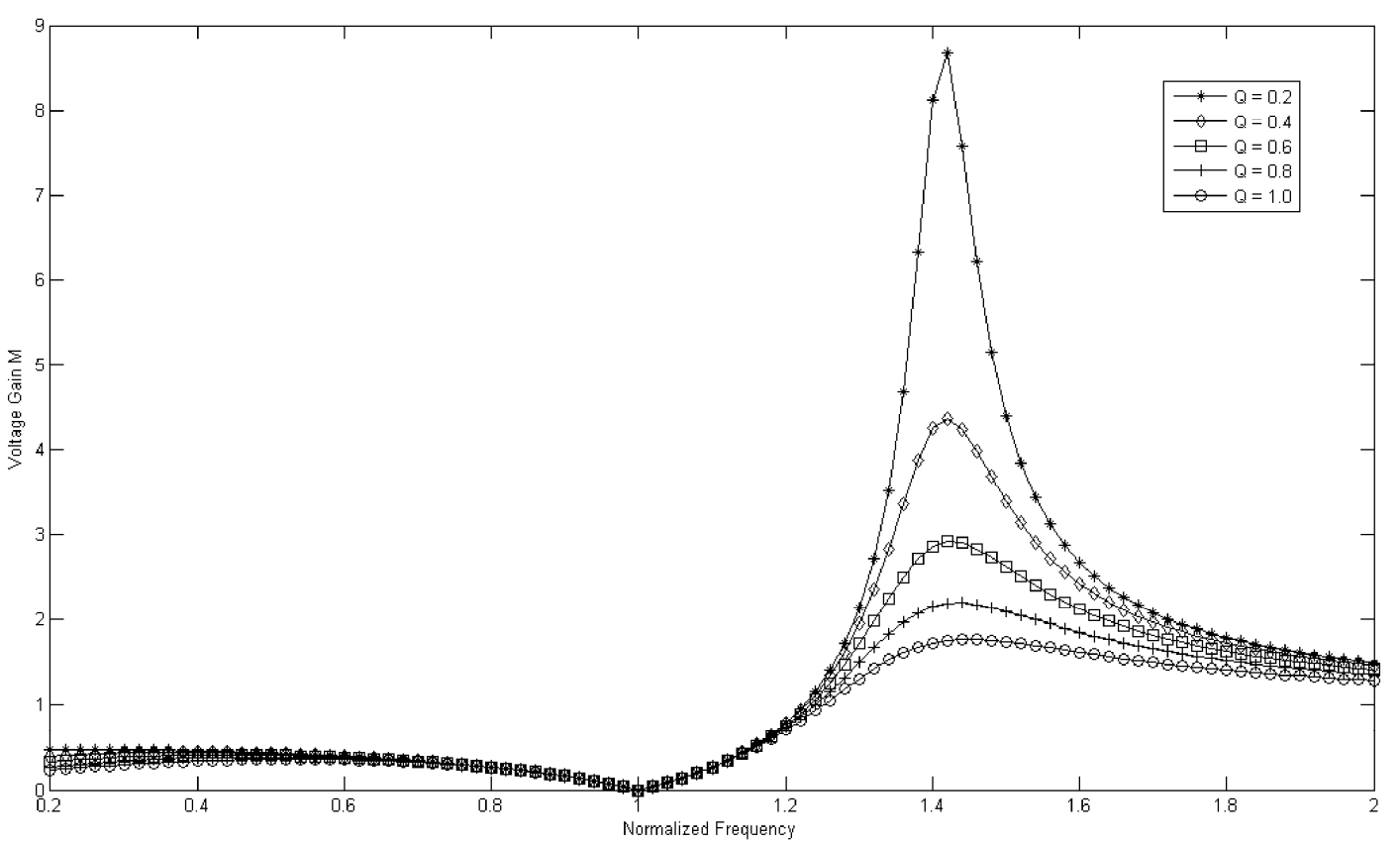

Figure 7. Voltage gain plot of topology 6

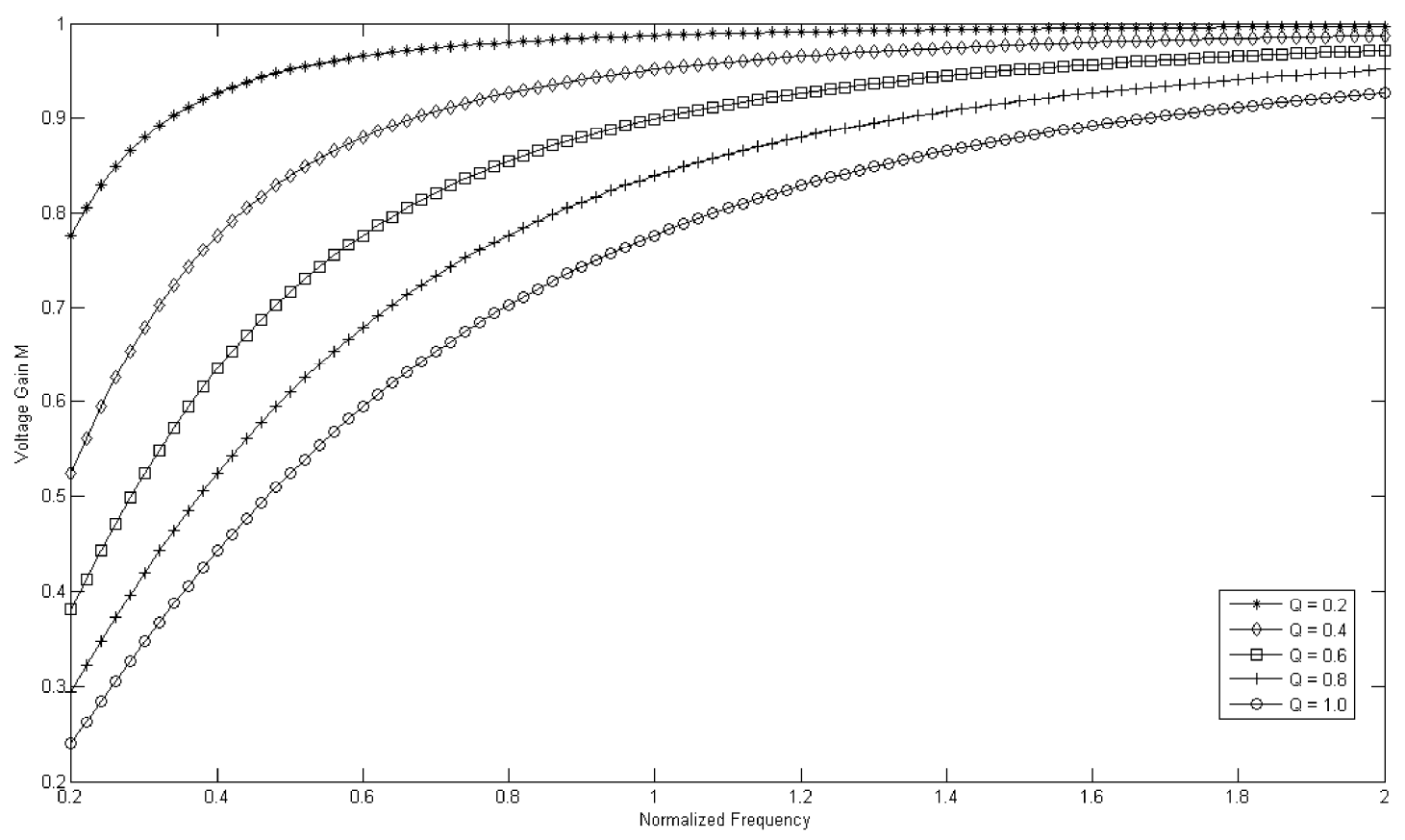

Figure 8 . Voltage gain plot of topology 8 


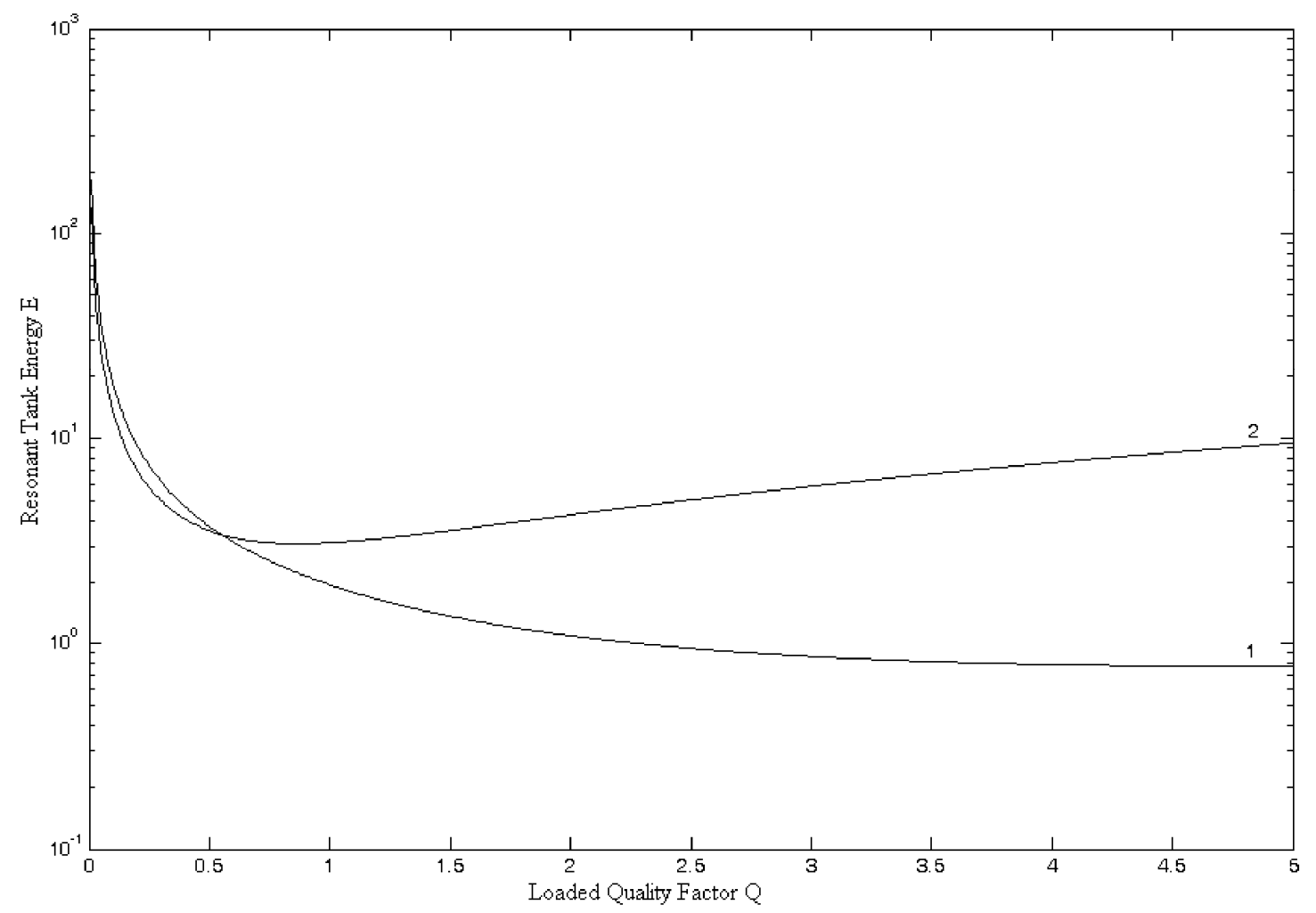

Figure 9. Energy vs Q for topology 1 and 2

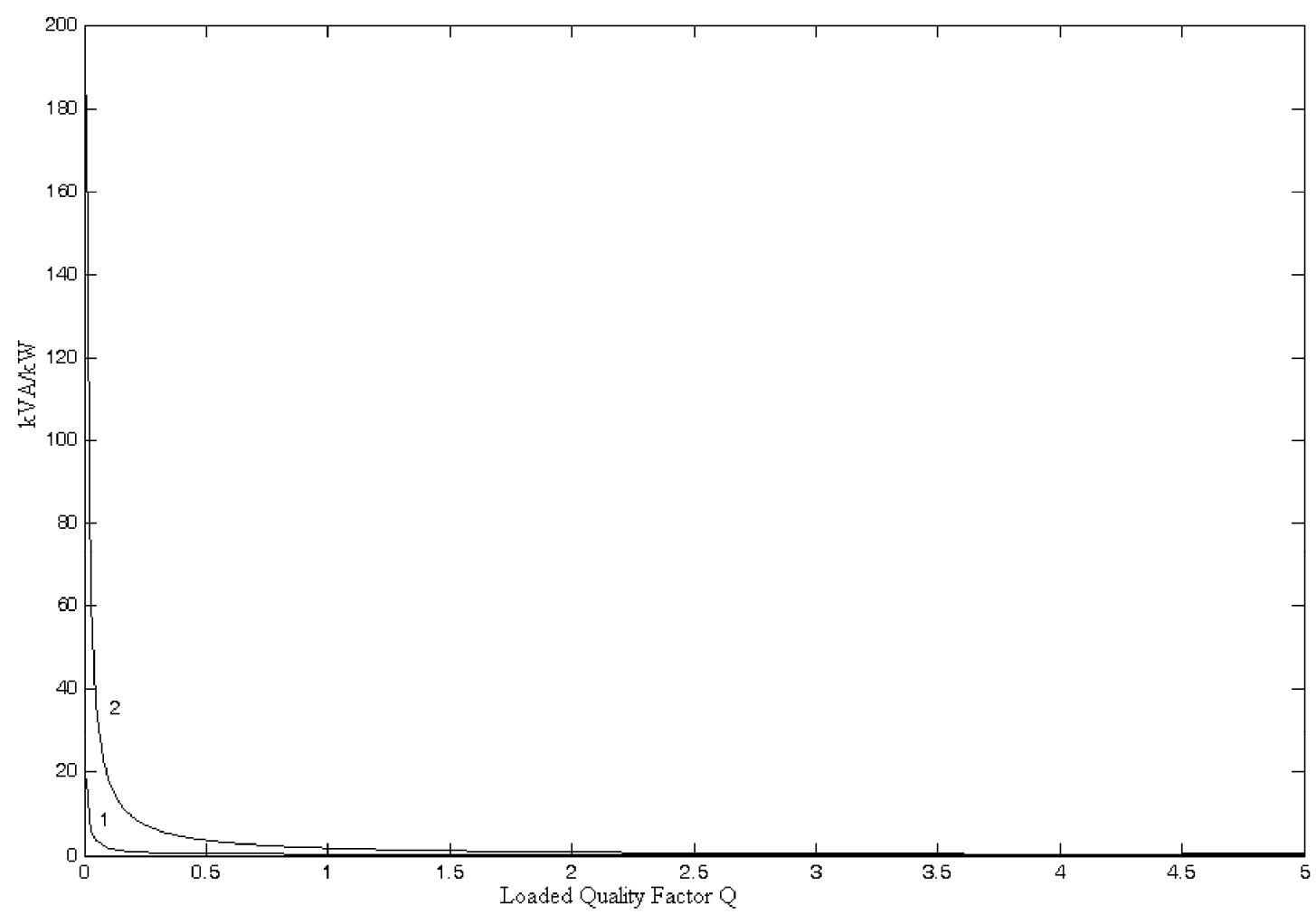

Figure 10. kVA/kW ratio plot for topology 1 and 2 